\title{
Trunnionosis and metal wear causing metal hypersensitivity: 2 sides of the same coin?
}

Jans van der Merwe, MBChB

Accepted January 7, 2021

\section{Correspondence to:}

J. van der Merwe

Department of Orthopaedic Surgery

University of Saskatchewan

LCD Main Box 99H, RR 3

Saskatoon SK S7K 3J6

jvvdmerwe@me.com

Cite as: Can J Surg 2021 October 13; 64(5). doi: 10.1503/cjs.013420

\section{SUMmARY}

Metal hypersensitivity (MHS) and trunnionosis are being looked at more frequently. Both entities pose a difficult concern for surgeons and patients alike. This commentary highlights the similarities and differences between the 2 conditions. When a surgeon suspects either MHS or trunnionosis, both should be considered in the differential diagnosis. Both conditions are rare and should be considered a diagnosis of exclusion. The commentary proposes an outline on how to diagnose and treat the 2 entities.
$\mathbf{J}$ oint replacements do exceptionally well overall, but a small percentage of patients are dissatisfied with their surgery. This dissatisfaction has led to more research and further investigations to understand metal hypersensitivity (MHS) and trunnionosis better. Metal hypersensitivity represents the body's inflammatory response to the metal wear debris that can occur from the bearing surface with metal-on-metal bearings or at modular junctions (i.e., metal head on taper or modular necks at the stem interface).

Despite several differences between MHS and trunnionosis, there are distinct similarities in the presentation, diagnosis and treatment options available for the 2 conditions. Therefore, one might wonder if they should be seen as separate entities or if they should fall under an umbrella term and should be investigated and treated as such.

\section{WHAT DO WE KNOW?}

Both conditions are type IV hypersensitivity reactions. Metal implants can undergo some degree of corrosion. The metal ions released due to corrosion lead to an immune response that can cause soft tissue inflammation with subsequent periprosthetic damage, with similarities in both conditions. ${ }^{1,2}$ Aseptic lymphocytic vasculitis (ALVAL) has a similar histological appearance than a delayed hypersensitivity response, but most lymphocytes seen in ALVAL are B cells compared with MHS, which is a T cell-mediated response. Trunnionosis and MHS have very little metal debris, but the continued release of metal ions leads to an immunological response.

Risk factors have been proposed in both conditions and include taper design, alloy combinations, length of implantation, modularity and higher activity levels. Both MHS and trunnionosis tend to occur more frequently in females. This is thought to be the result of sensitization with jewellery before joint implantation. ${ }^{3}$ There are, however, some studies reporting higher rates of trunnionosis in males and in patients with elevated body mass index. Trunnionosis is limited to the hip joint, whereas MHS can occur in both the hip and knee.

The most commonly cited symptoms for both conditions include pain, swelling and limited range of motion. ${ }^{4}$ In some cases, a rash may occur in either condition. In most cases, patients do not have any obvious reasons for 
the aforementioned symptoms, which complicates diagnosing and treating them.

\section{WHAT WE DON'T KNOW}

The role of routine serological markers (C-reactive protein [CRP] and erythrocyte sedimentation rate [ESR]) is unknown in MHS and trunnionosis. Newer investigations $(\alpha$-defensin, polymerase chain reaction [PCR]) may start to play a more important role in the diagnosis of infections in these limited situations.

Metal ions can be elevated following a joint replacement and therefore may not be very useful in decision making. In addition, no standardized values have been adopted yet.

Preimplantation screening for a joint replacement with skin patch testing is very controversial at present. Currently it should be performed only in patients with an acquired history of metal allergy or sufficient concerns based on a review of the systems. ${ }^{3}$ Skin patch testing relies on subjective reading and cannot be solely used by clinicians for guidance on implant choices. A positive test does not necessarily mean patients will get MHS following implantation, and a good outcome is not guaranteed by using hypoallergenic components. ${ }^{1}$

Postimplantation testing is similarly controversial. Patients with metal implants will have a higher rate of positive tests than the general population owing to prosthesisinduced sensitization. Leucocyte transformation testing (LTT) is more sensitive but not readily available.

\section{Proposed Patient WORKUP}

Surgeons should obtain a detailed history, including a metal allergy history, preoperatively. If patients have a history of metal allergy and have chronic complications following an arthroplasty, skin patch testing or LTT can be considered. ${ }^{3}$ Positive results should be interpreted with caution, seeing that prosthesis-induced sensitization can occur. ${ }^{5}$

Serum markers (CRP and ESR) should be obtained routinely. This is mainly useful to exclude a periprosthetic joint infection.

Synovial aspirates should be performed with elevated serological markers. The aspirates should be sent for manual cell counts (automatic cell counts can be falsely elevated with metal debris), differential as well as anaerobic and aerobic cultures (preferably cultured for 14-21 d) and acid-fast bacilli (AFB) and fungal cultures.

Arthroscopic inspections can be attempted in both hip and knee replacements to look for metallosis and corrosion at the articulations of the implants. More importantly, biopsies can be obtained for histopathology and microbiology. This is to confirm the histological appearance of a delayed hypersensitivity reaction.
Radiographs should be obtained routinely to exclude other causes for failure. Subtle changes can be seen in trunnionosis with incipient failure of flexible stems with an oblique angle between the femoral head and the femoral neck taper and/or faint radiodensity around the hip capsule. In MHS, radiolucent lines can eventually occur around the prosthesis. Ultrasonography, metal artifact reduction sequence magnetic resonance imaging and computed tomography can be used to evaluate cystic or soft tissue masses if present.

Metal ions should be used only as an adjunct or in research settings, not solely in decision making regarding treatments for both MHS and trunnionosis. The trend of metal ions might be more useful for interpretation than the individual values at a single point in time.

Nonoperative treatment should be the gold standard in patients suspected to have MHS or trunnionosis. This includes nonsteroidal anti-inflammatories for pain and swelling, and steroids for cutaneous rashes.

Dermatological referral might be useful in patients with persistent rashes in conjunction with a metal implant. Once the decision is made to continue with revision surgery, patients should be counselled regarding realistic expectations. These revisions have a higher morbidity without long-term data demonstrating success.

It is imperative that these patients be revised in a tertiary centre. Hypoallergenic components should be used to eliminate metal ion release. A thorough débridement should be performed to debulk the metal particle concentration in the periprosthetic tissues.

By screening patients appropriately at the initial visit, before any surgery, the surgeon can act preventatively, avoiding potential implant failure later on. Only patients with a history of metal allergy could be considered to undergo preoperative screening with skin patch testing or LTT. This shouldn't be done routinely. Patients with a history of metal allergy should rather be counselled appropriately regarding the limited body of evidence in treatment options and success rates.

Using hypoallergenic components routinely in the general population sounds attractive, but the increased cost associated with using them and the small group of patients affected by the use of standardized components makes them less appealing.

\section{Conclusion}

Even though it is difficult to group MHS and trunnionosis under the same umbrella term owing to the small differences observed, the diagnosis and treatment options do have similarities, and patients can be treated by following the same algorithm. It is useful to always consider both conditions in the differential diagnosis for patients with undetermined pain, limited range of motion and swelling with unequivocal diagnostic tests. 
Affiliations: From the Department of Orthopaedic Surgery, University of Saskatchewan, Saskatoon, SK.

Competing interests: None declared.

Content licence: This is an Open Access article distributed in accordance with the terms of the Creative Commons Attribution (CC BYNC-ND 4.0) licence, which permits use, distribution and reproduction in any medium, provided that the original publication is properly cited, the use is noncommercial (i.e., research or educational use), and no modifications or adaptations are made. See: https://creativecommons. org/licenses/by-nc-nd/4.0/

\section{References}

1. Middleton S, Toms A. Allergy in total knee arthroplasty: a review of the facts. Bone foint 7 2016;98-B:437-41.

2. Pinson ML, Coop CA, Webb CN. Metal hypersensitivity in total joint arthroplasty. Ann Allergy Asthma Immunol 2014;113:131-6.

3. Richards LJ, Streifel A, Rodrigues JM. Utility of patch testing and lymphocyte transformation testing in the evaluation of metal allergy in patients with orthopedic implants. Cureus 2019;11:e5761.

4. Morwood MP, Garrigues GE. Shoulder arthroplasty in the patient with metal hypersensitivity. 7 Shoulder Elbow Surg 2015;24:1156-64.

5. Delimar D, Bohacek I, Paštar Z, et al. Orthopedic and cutaneous reactions to nickel after total hip replacement. Acta Dermatovenerol Croat 2018;26:39-43 